\title{
A Hybrid Cooperative Model for Bandwidth Utilization in Vehicular Ad hoc Networks Based on Game Theory
}

\author{
Esraa Eldesouky ${ }^{1}$, Walaa Elashmawi ${ }^{2}$, Li Renfa $^{1}$ and Wang Dong ${ }^{1}$ \\ ${ }^{1}$ College of Information Science and Engineering, Hunan University, 410082, \\ Changsha, China \\ ${ }^{2}$ Faculty of Computers and Informatics, Suez Canal University, Ismailia 41522, \\ Egypt \\ esraa_m@hnu.edu.cn
}

\begin{abstract}
Generally speaking, Vehicular Ad hoc Networks comprise vehicles and roadside stations as the common types of nodes. These nodes are mainly providing each other with relevant information. A wide range of applications are supported by Vehicular Ad hoc Networks such as file downloading, or access to the Internet with lack of resource controlling. These challenging resource managements degrade the network performance and create competitive environments. Through this paper, we introduce a novel algorithm for bandwidth sharing enhancement in both vehicle-to-vehicle and vehicle-to-roadside communications. We aim to improve the bandwidth usage based on coalition formation game in order to reduce the cost paid by vehicles for bandwidth reservation at roadsides. Mainly, we intend the enhancement of both individual vehicles' payoff along with the whole network gain. Simulation results have proven that the proposed algorithm succeed to analyze this conflicting situation efficiently. In addition, satisfaction rates up to $75 \%$ have been achieved with an incredibly increase in the network throughput.
\end{abstract}

Keywords: Vehicular Ad hoc Networks; Game Theory; Vehicle-to-Roadside Communications; Bandwidth Sharing; Data Transfer Rate

\section{Introduction}

Vehicular Ad hoc Networks (VANETs) is a technology whereas vehicles and roadsides are employed to create an intelligent network [1]. Each vehicle can be considered as a node or router that can support several Intelligent Transportation Systems (ITS) applications. The IEEE 802.11p and IEEE 1609 standards is adopted to utilize the Dedicated Short Range Communication (DSRC) wireless protocol specifically designed for VANETs $[2,3]$. It is used to support short and medium ranges of communications including Vehicle-to-Vehicle (V2V) and Vehicle-to-Roadside (V2R) communications. Despite safety requirements, DSRC allows VANETs to provide public services like content sharing, email browsing, or internet access.

The diversity of applications in VANETs leads to basic problems due to mobility, resource limitations, and unbalanced distribution. As a result, network throughput is reduced and a competitive environment is created [4]. In wireless networks, bandwidth is defined as "the maximum limits for data transfer over a wireless spectrum" [5]. So far, the increasing needs to frequently access data and limitation of the available resources can result in a conflicting situation.

In this paper, we propose a hybrid algorithm for bandwidth sharing by utilizing both $\mathrm{V} 2 \mathrm{~V}$ and V2R communications. Based on coalition formation game, such conflicting situation can be avoided by forming cooperative sets (i.e. Coalitions) among the vehicular nodes and roadsides within their vicinity [6]. Vehicular nodes are considered to be game players that are seeking to gain the highest individual profits. However, we are also 
concerned with attaining better data transfer rates in parallel with decreasing the cost of using this available bandwidth.

The paper is organized as follows: Section 2 summarizes the literature work. In Section 3 , we explain our conflicting problem and calculate the cost and utility. The proposed algorithm is presented in Section 4. Section 5 includes the performance measures and the experimental results. Finally, we conclude our paper in Section 6.

\section{Related Work}

Game theory, as a powerful tool, attracts several researchers to resolve numerous competitive issues over wireless networks. In [7, 8], authors explained the basic concept and the way to analyze any conflicting situation in wireless network in general. They afforded a reference for how to formulate a utility function based on the addressed problems, such as power usage and resource management. In wireless networks, these problems have been in investigated in a number of research works [9-12].

Bandwidth sharing problems in VANETs have been studied in different researches. A proxy-based V2R access protocol that supports collaborative packet forwarding among vehicles and roadsides is introduced [13]. This protocol aims to reduce contention among vehicular nodes by aggregating packets to some selected vehicles called proxies, which improves the system performance. However, authors proposed a cross-layer protocol for peer communication among vehicular nodes to enhance the data transfer rates [14]. Based on the nearest base stations, vehicles can get the requested services via a multi-hop route. Although [13] and [14] target the overall network output, they never consider the individual profits for vehicular nodes.

In [15], the first coalition formation game approach for V2R communication is presented. The proposed approach considers the cooperation among vehicular nodes to enhance the social welfare. Another coalition formation model is proposed in [16] for maximizing the bandwidth allocation in VANETs. This model is only relying on the intervehicle communications without considering the presence of any infrastructure.

In this work, we are concerned with the bandwidth utilization issues as a limited sharable resource. Although our proposed model and the work introduced in [15] target the vehicular users' behaviors for bandwidth utilization, but the model introduced in [14] considers only 9 roadsides and 4 users. Contrary to [14], the proposed algorithm is based on IEEE 802.11p standard [17] instead of IEEE 802.16 standard, which is mainly intended to VANETs. Unlike [13-15], we consider the enhancement of the individual profits as well as the overall network throughput. Finally compared to [16], our primary goal is to study the cooperation among vehicles and roadsides in order to achieve a supportive and collaborative environment.

\section{The System Model}

In VANETs, the variety of the provided intelligent applications and services creates a wide range of exchangeable data including safety and content information. As a Consequence, conflicting and competitive environments are produced due to the existence of selfish vehicular nodes that seek for collecting the demanded bandwidth. In this paper, we use the coalition formation game for its ability to resolve conflicting situations and enhance the individual profit as well [6].

Through our model, we presume that vehicles are supplied by suitable communication devices to ensure both V2V and V2R communications. These devices helps vehicular nodes to access the IEEE 802.11p wireless spectrum. Moreover, additional sensors are mounted to these nodes such as Global Positioning System (GPS) devices. This competitive environment is simulated using the proposed model in order to achieve better bandwidth distributions and improve the data transfer rate. We formulate our model based on coalition formation as an Input-Output $(I / O)$ algorithm, in which $I$ is the set of 
vehicles looking for the demanded bandwidth, $O$ is the formed feasible coalitions, and $P$ is the processing operation. The processing operation mimics the efficient bandwidth sharing as to enhance the overall payoff.

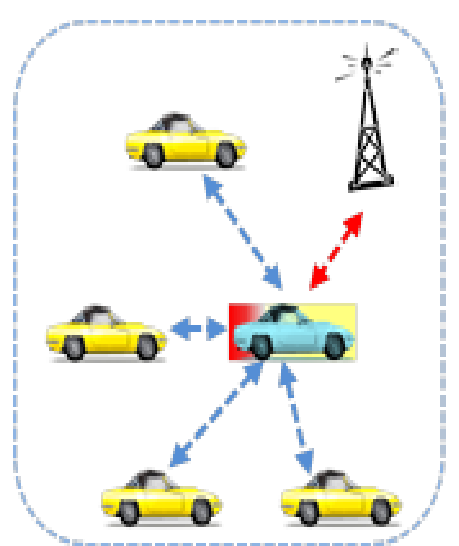

(a)

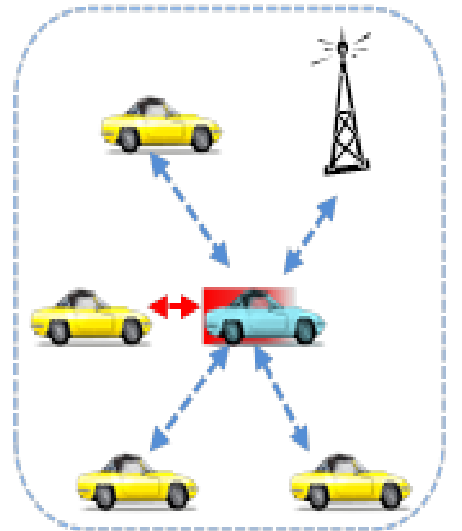

(b)

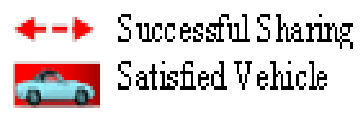

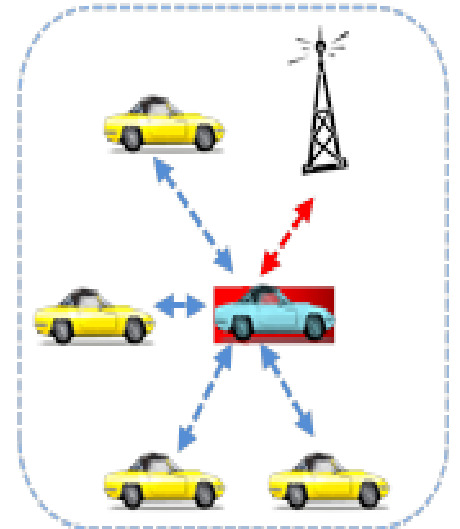

(c)

Figure 1. Coalition Formation Graph for a Demanded Vehicle

According to Figure 1, a demanded vehicular node seeks for the needed bandwidth in order to be able to send or receive data (e.g., downloading content or streaming a video). During its journey, it starts to form coalition with surrounding vehicles and roadsides, although, the highest priority for reserving bandwidth is always given to the nearest roadside. The reason behind this is based upon the premise of the cheapest unit cost price being offered by the roadside, compared to a vehicle, and the high probability of the bandwidth availability at the specified roadside. However, if this vehicle is unable to complete the requested bandwidth, it asks for cooperation from other vehicles within its vicinity with higher cost price. Finally, the vehicle is satisfied if only, it succeeds to get all the demanded bandwidth for accessing its data.

\subsection{Model Formulation}

Mainly, we are concerned with the balanced distribution of the bandwidth among vehicles in the roadside transmission range. In this model, we consider a set of vehicular nodes $V=\left\{v_{1}, v_{2}, \ldots, v_{n}\right\}$, a set of installed roadsides $R=\left\{r_{1}, r_{2}, \ldots, r_{m}\right\}$, and a set of nonoverlapping wireless channels $W c=\left\{w_{1}, w_{2}, \ldots, w_{l}\right\}$. Vehicular nodes use these DSRC channels in order to directly communicate with each other. Our main target is to achieve the cooperation among vehicular nodes as to enhance the bandwidth allocation and thus improve the data transfer rate.

Given any vehicle $\left(v_{i}\right)$ can join a coalition $\left(c_{y}\right)$ in order to fulfill the requested bandwidth $\left(B_{t}\right)$ given that the coalition set $(C)$ is defined as $C=\left\{c_{1}, c_{2}, \ldots, c_{x}\right\}$ and $y=1,2, \ldots, x$. Vehicular nodes can form a set of $(x)$ coalitions with the installed roadsides and vehicles, where $\left(x \in 2^{n}\right)$. Any coalition $\left(c_{y}\right)$ is a non-empty set which contains vehicles $(V)$ and roadsides $(R)$. In our algorithm, we assume that for any vehicle $\left(v_{i}\right)$ can be either bandwidth requester or giver, however any roadside $\left(r_{j}\right)$ can only supply the needed bandwidth.

\subsection{Cost and Utility}


A vehicle $\left(v_{i}\right)$ can join any coalition $\left(c_{y}\right)$ in an effort to get the necessary bandwidth. Nevertheless, this vehicle will endure a calculated cost based on the channel accessed $\left(w_{k}\right)$. The cost price is defined as $\zeta_{v i}\left(c_{y}, w_{k}\right) \in \square^{+}$and is calculated as in equation (1):

$$
\zeta_{v i}\left(c_{y}, w_{k}\right) \in \square^{+}= \begin{cases}R_{\theta} * B_{t} & \forall N l_{v i} \in R \\ & \forall N l_{v i} \in V\end{cases}
$$

Where $N l_{v i}$ is the direct neighbor list of the requested vehicle $\left(v_{i}\right)$, and $R_{\theta}$ is the price unit which is computed as $R_{\theta}=0.3 / \rho$ for $\rho$ is the transmission rate per one unit of bandwidth $\left(B_{t}\right)$, given that $\rho=27 \mathrm{KBps}$. Additionally, the price unit $\left(V_{\theta}=0.3 / \varepsilon\right)$ determine the incurred cost due to demanding bandwidth from a vehicle, then according to $[18,19], \varepsilon$ is set as $16.67 \mathrm{KBps}$.

Generally, each vehicular node taking part in the coalition formation game vies for a share value $S h_{v i} \in \square^{+}$, which is gained by allocating the requested bandwidth for each wireless channel. This share value is referred to as the maximum bandwidth that can be allocated from each channel. According to IEEE $802.11 \mathrm{p}$ standard, each channel is defined to have $10 \mathrm{MHz}$ as the maximum bandwidth [20]. Vehicles aim to maximize their own profit as well as the overall network throughput. Therefore, with bandwidth allocation in coalition $\left(c_{y}\right)$, the vehicle's utility $\left(U T_{v i}\right)$ is defined to be:

$$
U T_{v i}=S h_{v i}-\zeta_{v i}\left(c_{y}, w_{k}\right)
$$

The participating vehicular nodes are motivated to form different coalitions in order to enhance the individual profits as well as reducing the cost paid through finding the feasible coalition ( $C F^{*}$ ). According to equation (3), we aim to maximize the total gained revenue as follows:

$$
C F^{*}=\arg \max \sum_{i=1}\left(U T_{i}\right)
$$

\section{V2R Coalition Formation Algorithm}

Communications in VANETs are used for supporting vehicular nodes with several intelligent applications and services [18]. In this section, we introduce a new algorithm for improving the network scalability and robustness based on achieving cooperative VANETs for bandwidth sharing. We intend to fairly use the available bandwidth in parallel with enhancing the data transfer rates.

As mentioned above, vehicles can form coalition with vehicular nodes within their transmission ranges to get the requested bandwidth. This can be accomplished by applying the following algorithm: 


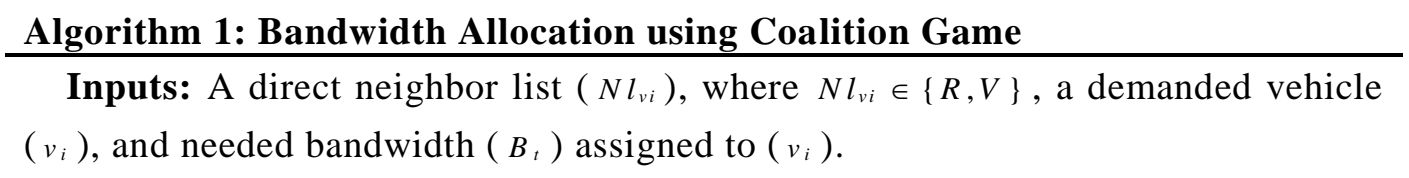

Output: The formed coalition $\left(C F^{*}\right)$ with the highest total revenue Processing:

1. Add $\left(v_{i}\right)$ to the empty coalition $\left(c_{y}\right)$, such that $\left(c_{y}=\left\{v_{i}\right\}\right)$

2. Search in $\left(N l_{v i}\right)$

Case 1: $\forall N l_{v i} \in R$

If $\left(B t_{v i}<B_{-} R_{j}\right)$, then

a. Calculate the remaining bandwidth $\left(B t_{v i}\right)$ for $\left(v_{i}\right)$.

b. Add this neighbor to the coalition $\left(c_{y}\right)$, where $\left(c_{y}=c_{y} \cup N l_{v i}\right)$

c. Compute the cost $\zeta_{v i}\left(c_{y}, w_{k}\right)$ and utility $\left(U T_{v i}\right)$ using equation (1) and (2) respectively.

d. Go to Step 2.

\section{Else}
a. Update the neighbor's bandwidth $\left(B_{-} R_{j}\right)$
b. Add this neighbor to the coalition $\left(c_{y}\right)$, where $\left(c_{y}=c_{y} \cup N l_{v i}\right)$
c. Compute the cost $\zeta_{v i}\left(c_{y}, w_{k}\right)$ and utility $\left(U T_{v i}\right)$ using equation (1) and (2) respectively.
d. Go to Step 3.

\section{End If}

Case 2: $\forall N l_{v i} \notin R$

If $\left(B t_{v i}<B{ }_{-} N_{v i}\right)$, then

a. Calculate the remaining bandwidth $\left(B t_{v i}\right)$ for $\left(v_{i}\right)$.

b. Add this neighbor to the coalition $\left(c_{y}\right)$, where $\left(c_{y}=c_{y} \cup N l_{v i}\right)$

c. Compute the cost $\zeta_{v i}\left(c_{y}, w_{k}\right)$ and utility $\left(U T_{v i}\right)$ using equation (1) and (2) respectively.

d. Go to Step 2.

Else

a. Update the neighbor's bandwidth $\left(B_{-} N_{v i}\right)$

b. Add this neighbor to the coalition $\left(c_{y}\right)$, where $\left(c_{y}=c_{y} \cup N l_{v i}\right)$

c. Compute the cost $\zeta_{v i}\left(c_{y}, w_{k}\right)$ and utility $\left(U T_{v i}\right)$ using equation (1) and (2) respectively. 


\section{d. Go to Step 3.}

\section{End If}

3. Stop Search and Return the maximum utility Coalition $C F$ *

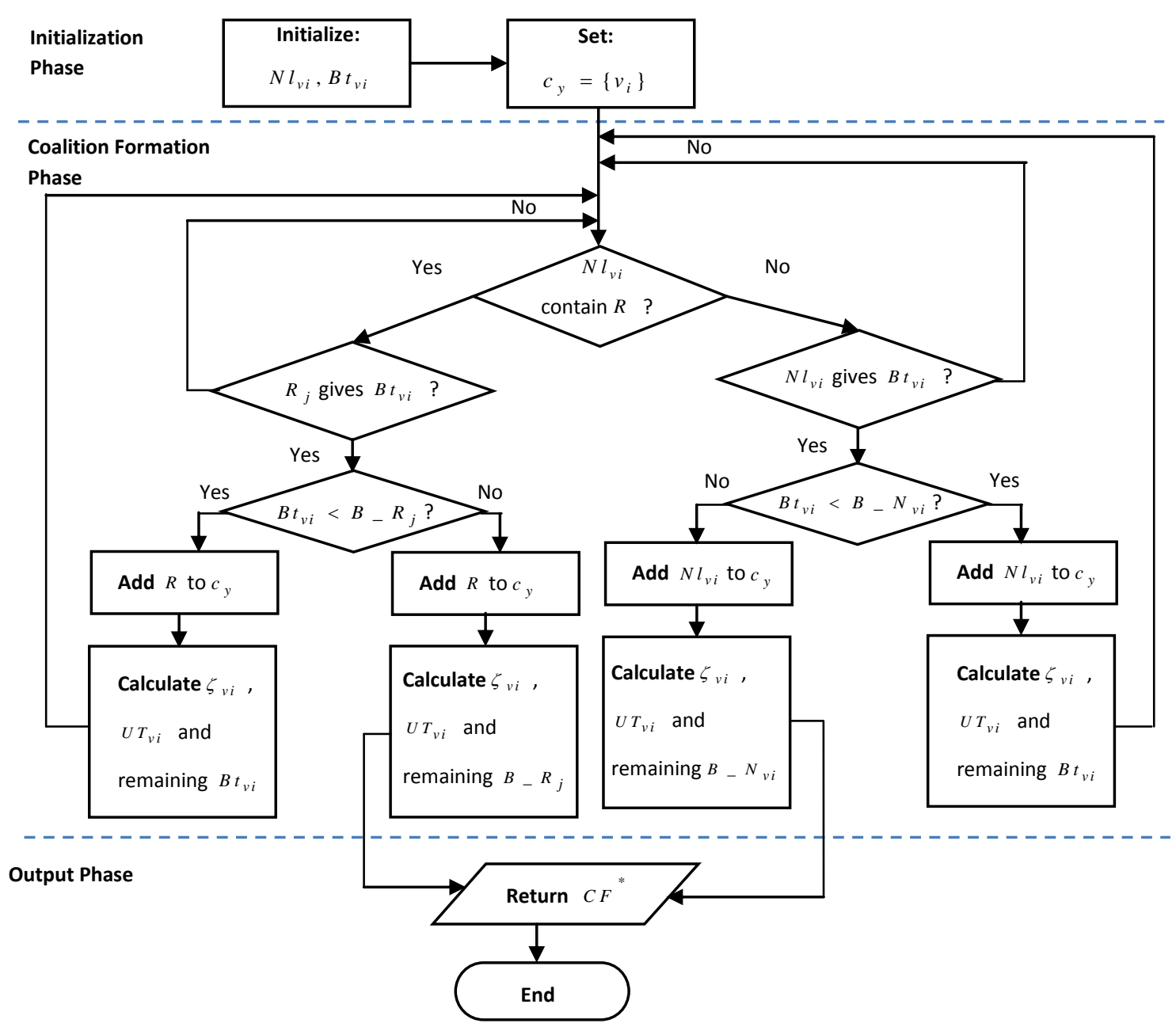

Figure 2. Coalition Formation Algorithm Flowchart Diagram

According to the proposed algorithm, vehicular nodes can form cooperative coalitions with their direct and indirect neighbors within their coverage areas. However, a demanded vehicular node commences by searching its nearest neighbor list. The main target is to find a roadside in the neighbor list with the highest probability of sharing its bandwidth. Nevertheless, if the roadside's resource is not enough to satisfy the demanded vehicle's needs, the algorithm continues to include the indirect neighbors till this node's requirement is fulfilled. There may exist a case where there is no roadside in the direct neighbor list of the demanded vehicular node. In this situation, the vehicular node searches for needed bandwidth within the nearest vehicles with higher cost price. Finally, this algorithm stops when the demanded vehicular node is satisfied. An illustrative flow chart for our algorithm is shown in Figure 2. 


\section{Experiment Criteria and Results}

\subsection{Performance Criteria}

The accuracy and efficiency of the proposed model is examined through several experiments. Each experiment has a defined set of vehicles and roadsides, besides predetermined channels are assigned to this set with the requested bandwidth. Moreover, Eclipse Java EE IDE is used to implement our model on a machine with the following specification, including: $2.2 \mathrm{GHz}$ CPU unit, $8 \mathrm{~GB}$ for the memory, and Window 7 (64 bits). As mentioned above, our model aims to enhance the bandwidth distribution among competing vehicular nodes to improve the data transfer rates.

Different numbers of vehicles are tested accompanied with some roadsides as shown in Table 1. However, we are not able to increase the examined population for more than 5o vehicular nodes, as we are limited to specified machined configurations.

Table 1. Simulated Environments

\begin{tabular}{|c|c|c|c|}
\hline $\begin{array}{c}\text { Experiment } \\
\text { Number }\end{array}$ & $\begin{array}{c}\text { Number of } \\
\text { Vehicular nodes }\end{array}$ & $\begin{array}{c}\text { Roadside } \\
\text { Density }\end{array}$ & $\begin{array}{c}\text { Number of } \\
\text { Direct Neighbors }\end{array}$ \\
\hline 1 & 10 & 1 & 2 \\
\hline 2 & 20 & 3 & 4 \\
\hline 3 & 30 & 5 & 6 \\
\hline 4 & 40 & 7 & 8 \\
\hline 5 & 50 & 9 & 10 \\
\hline
\end{tabular}

We applied several experiments on distinct vehicles' density for examining the performance of the proposed model. Each of the presented experimented is implemented for 100 times and the average was calculated (see Table 1). Unlike [16], we targeted V2V and V2R communications environment as to simulate the vehicular node natural actions. Moreover, we succeeded to mimic large number of vehicular nodes compared to the work proposed in [15, 19].

We present some evaluation criteria in order to study the efficiency of the proposed algorithm. The average number of the roadsides which join the feasible coalitions is calculated according to the following equation:

$$
A v g_{-} R S=\left(\sum_{i=1}^{R S_{-} t} P a r_{-} R S_{i}\right) / R S_{-} t
$$

Where $R S_{-} t$ is the total number of the installed roadsides, and $\operatorname{Par}_{-} R S_{i}$ is the number of the roadsides participated in the coalition formation game. However in equation (5), we measure the average satisfaction gain obtained from forming $l$ coalitions and is computed as follows:

$$
V e h_{-} U=\sum_{i=1}^{l}\left(U T_{i}\right)
$$

The vehicles' satisfaction percentage represents the average number of vehicular nodes which are succeeded to collect the requested bandwidth $(x)$ in comparable with the total number of the demanded vehicles $\left(R Q_{-} t\right)$. This measurement is calculated according to equation (6):

$$
S P=\sum_{i=1}^{R Q_{-} t}\left(x_{i}\right) / R Q_{-} t
$$


Finally, the average Data Transfer Rate $\left(A V G_{-} T R\right.$ ) evaluates the successful bandwidth allocation for the requested channels (as in equation 7).

$$
A V G_{-} T R=\sum_{j=1}^{l} \sum_{i=1}^{R E Q_{c h}} Y_{i j} / \text { time }
$$

Where $l$ total number of wireless channel, $R E Q_{-} c h$ the total requested channels, $Y$ is the successful allocated vehicles, and time is the total time required in order to achieving the targeted goal.

\subsection{Simulation Result and Analysis}

According to the conducted experiments in Table 1, our simulation results are analyzed as following. Figure 3 illustrates the result of applying the evaluation metric calculated using equation (4).

It is the average ratio of the roadsides that positively join coalitions and supply vehicular nodes with the requested bandwidths. However, due to the fluctuations of the result according to vehicles and roadsides, more future work will be conducted to investigate the reason behind this.

Demanded vehicles, which succeed to form feasible coalitions, obtained an average utility numerical results stated in Figure 4. As shown, the results of using $\mathrm{V} 2 \mathrm{~V}$ approach are higher than those gained from using V2R approach. This occurred because the presence of roadsides in the V2R approach reduces the unit cost as well as supply vehicles with higher bandwidths; therefore, a few numbers of coalitions is obtained. Nevertheless, the utility results are incrementing according to the number of vehicles in both approaches. Consequently, the individual vehicles benefits a long with the overall network welfare are not affected.

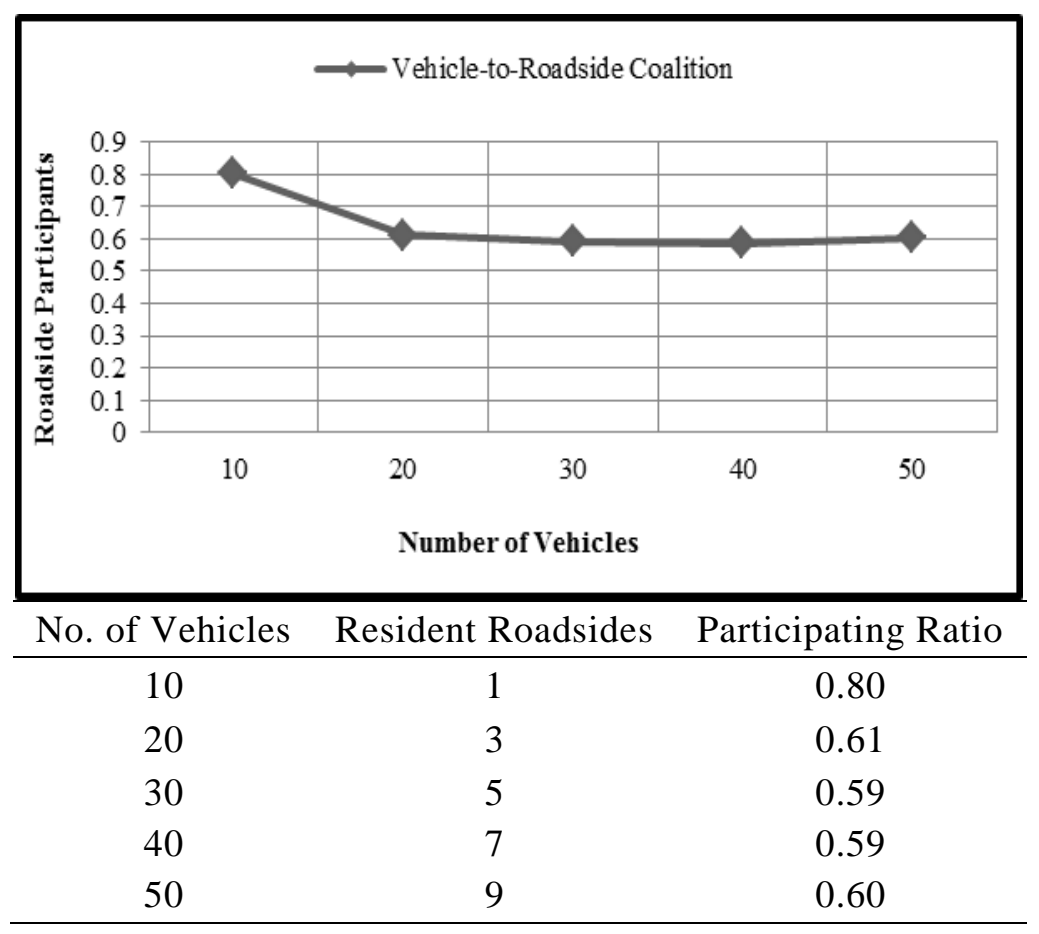

Figure 3. The Average Ratio of the Participating Roadsides 


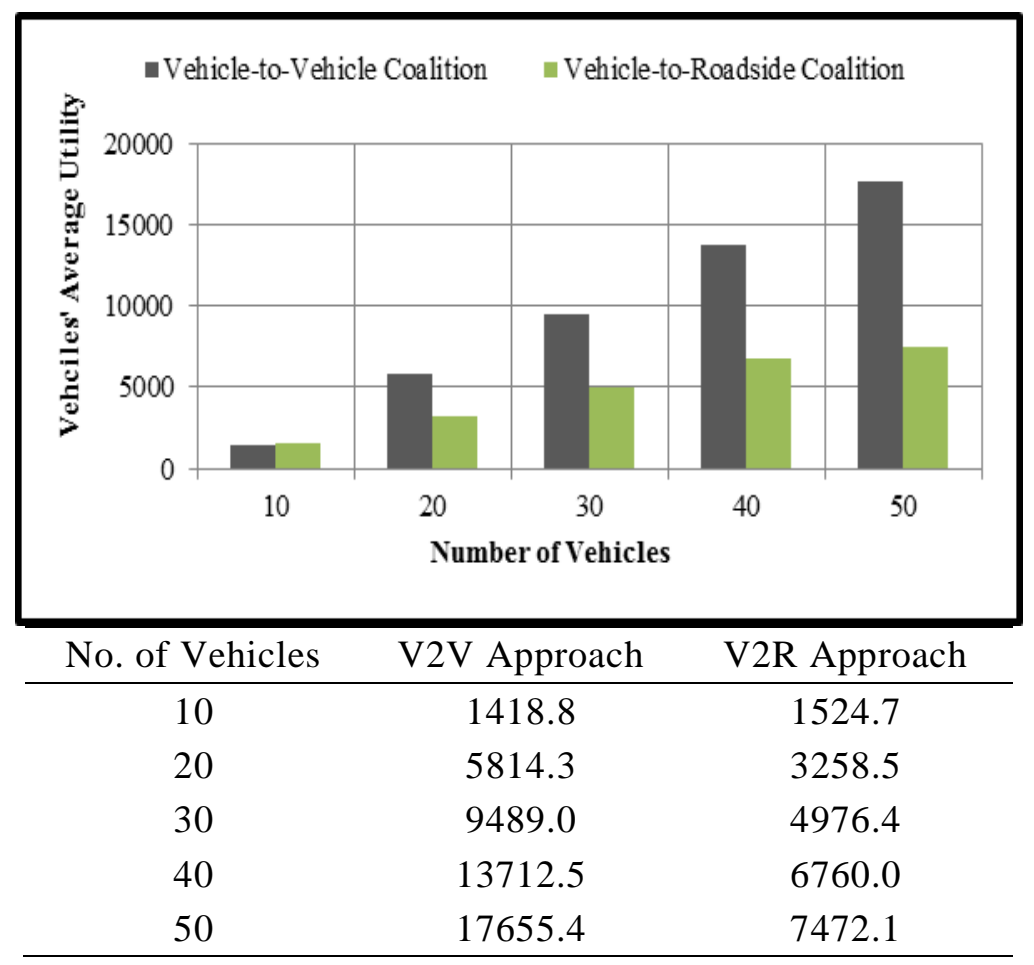

Figure 4. The Average Total Revenue Resulted from Forming Coalitions

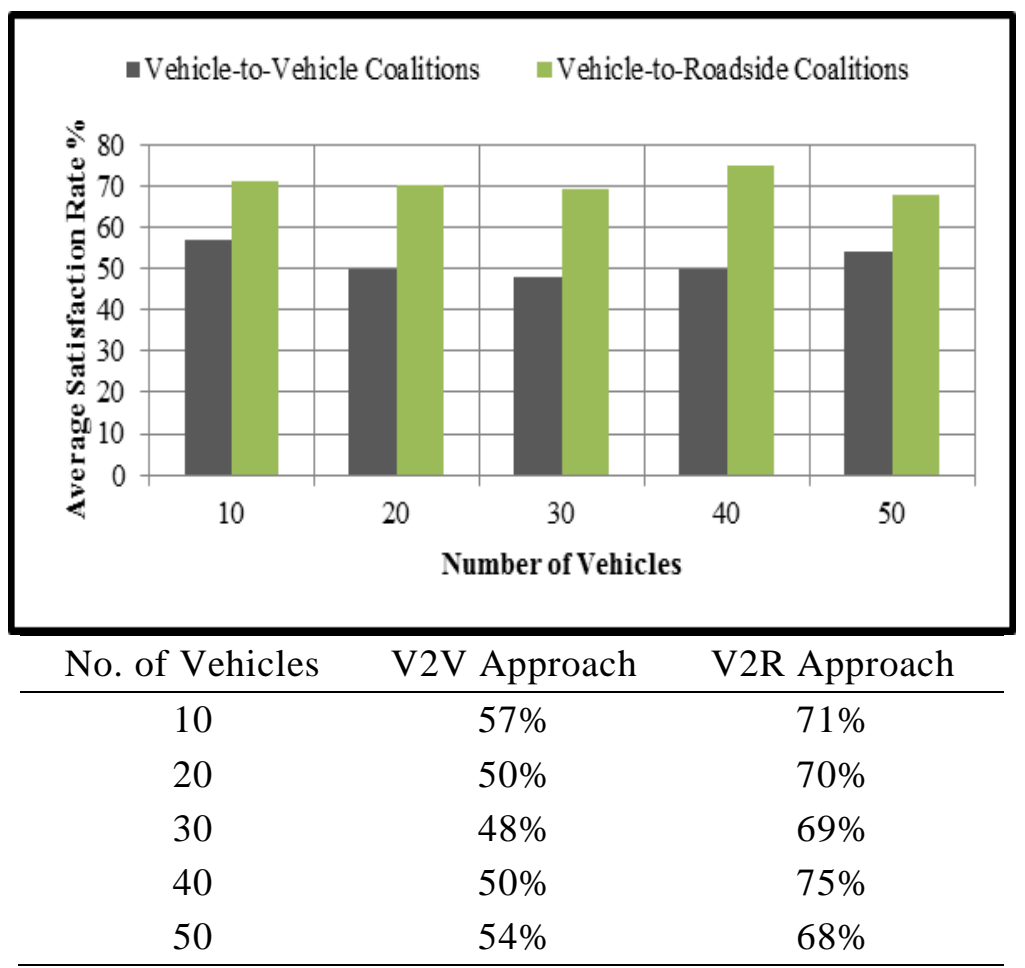

Figure 5. The Satisfaction Rate (\%) of the Proposed Model 


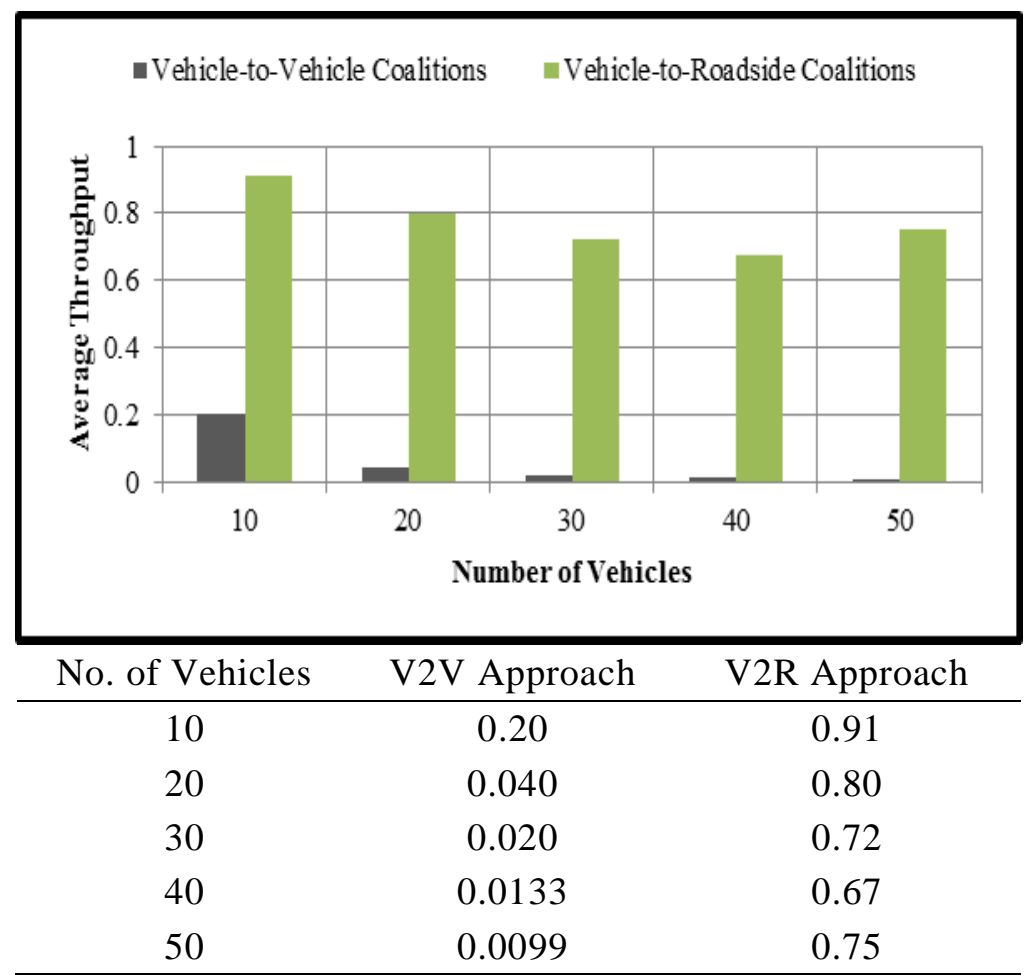

\section{Figure 6. Simulation Results of the Data Transfer Rates in Mbps}

The average satisfaction ratios for the demanded vehicular nodes over different environments are illustrated in Figure 5. Vehicular nodes are able to utilize the limited bandwidth with an approximately average satisfaction of $71 \%$ for the V2R approach. According to the attached numerical analysis, the maximum ratio that we recorded was $75 \%$ for 40 vehicular nodes contending to access the available 7 roadsides. Compared to V2V approach, the average satisfaction rate was only $52 \%$. As a result, the presence of roadsides is essential to accomplish higher data transfer rates.

Vehicular nodes can communicate with the roadsides as to improve the data transfer rates as well as the range of communication [1]. Based on Figure 5, nodes which rely on roadsides in order to cache the demanded bandwidth, succeeded to achieve an incredible higher data rates.

Finally, the average throughput is shown in Figure 6. For the V2R approach, vehicles are able to get extremely higher throughput compared to the $\mathrm{V} 2 \mathrm{~V}$ approach. A maximum throughput of approximately $0.911 \mathrm{Mbps}$ was reached for vehicular density of 10 vehicles. Moreover, the relationship among vehicles population and data transfer rates is inversely proportional over $\mathrm{V} 2 \mathrm{~V}$ communications. The reason behind this result is that, as more vehicles are demanding more bandwidth amounts, the network workload is increased and consequently reduces the throughput.

\section{Conclusion}

Several challenges arise regarding the bandwidth sharing problems and their fair distribution among vehicles in VANETs due to fragile connectivity and high mobility. These nodes are always seeking for caching and improving their data transmission rates. Through the proposed work, we introduced a bandwidth sharing model based on coalition formation game theory in a vehicle-to-roadside environment. This model has proved to significantly improve the data transfer rates with a maximum throughput of 0.91 Mbps over all the examined areas. 
Additionally, according to our simulation results, vehicular nodes are stimulated to join the feasible coalitions as to increase their individual payoffs (i.e., maximize bandwidth rates) in conjunction with decreasing the cost of reserving the needed bandwidths. However, numerous future works should consider unexplored issues such as roadside-to-roadside cooperation as to fulfill the vehicles requirements. Moreover, handling the channel radios to reduce the wasted bandwidth should be studied.

\section{Acknowledgements}

This work was supported by National Key Technology R\&D Program of China (2012BAD35B06).

\section{References}

[1]. S. Al-Sultan, M. M. Al-Doori, A. H. Al-Bayatti and H. Zedan, "A comprehensive survey on vehicular Ad Hoc network," Journal of network and computer applications, vol. 37, (2014), pp. 380-392.

[2]. I. W. Group, "IEEE Standard for Information Technology-Telecommunications and Information Exchange between Systems-Local and Metropolitan Area Networks-Specific Requirements-Part 11: Wireless LAN Medium Access Control (MAC) and Physical Layer (PHY) Specifications Amendment 6: Wireless Access in Vehicular Environments," IEEE Std, vol. 802, (2010), pp. 11.

[3]. R. Uzcategui and G. Acosta-Marum, "Wave: a tutorial," Communications Magazine, IEEE, vol. 47, (2009), pp. 126-133.

[4]. D. Wu, Y. Ling, H. Zhu and J. Liang, "The RSU access problem based on evolutionary game theory for VANET," International Journal of Distributed Sensor Networks, vol. 2013, (2013).

[5]. S. Yousefi, M. S. Mousavi, and M. Fathy, "Vehicular ad hoc networks (VANETs): challenges and perspectives," in ITS Telecommunications Proceedings, 2006 6th International Conference on, (2006), pp. 761-766.

[6]. Z. Khan, S. Glisic, L. A. DaSilva, and J. Lehtomaki, "Modeling the dynamics of coalition formation games for cooperative spectrum sharing in an interference channel," Computational Intelligence and AI in Games, IEEE Transactions on, vol. 3, (2011), pp. 17-30.

[7]. A. B. MacKenzie and S. B. Wicker, "Game theory in communications: Motivation, explanation, and application to power control," in Global Telecommunications Conference, 2001. GLOBECOM'01. IEEE, (2001), pp. 821-826.

[8]. D. E. Charilas and A. D. Panagopoulos, "A survey on game theory applications in wireless networks," Computer Networks, vol. 54, (2010), pp. 3421-3430.

[9]. D. Niyato and E. Hossain, "Queue-aware uplink bandwidth allocation and rate control for polling service in IEEE 802.16 broadband wireless networks," Mobile Computing, IEEE Transactions on, vol. 5, (2006), pp. 668-679.

[10].D. Niyato and E. Hossain, "A queuing-theoretic and optimization-based model for radio resource management in IEEE 802.16 broadband wireless networks," Computers, IEEE Transactions on, vol. 55, (2006), pp. 1473-1488.

[11].D. Niyato and E. Hossain, "A cooperative game framework for bandwidth allocation in 4G heterogeneous wireless networks," in Communications, 2006. ICC'06. IEEE International Conference on, (2006), pp. 4357-4362.

[12].S. Vassaki, A. D. Panagopoulos, and P. Constantinou, "Bandwidth allocation in wireless access networks: Bankruptcy game vs cooperative game," in Ultra Modern Telecommunications \& Workshops, 2009. ICUMT'09. International Conference on, (2009), pp. 1-4.

[13].M.-F. Jhang and W. Liao, "Cooperative and opportunistic channel access for vehicle to roadside (V2R) communications," Mobile Networks and Applications, vol. 15, (2010), pp. 13-19.

[14].K. Yang, S. Ou, H.-H. Chen and J. He, "A multihop peer-communication protocol with fairness guarantee for IEEE 802.16-based vehicular networks," Vehicular Technology, IEEE Transactions on, vol. 56, (2007), pp. 3358-3370.

[15].D. Niyato, P. Wang, W. Saad and A. Hjorungnes, "Coalition formation games for bandwidth sharing in vehicle-to-roadside communications," in Wireless Communications and Networking Conference (WCNC), 2010 IEEE, (2010), pp. 1-5.

[16].E. Eldesouky, W. Elashmawi, W. Dong and L. Renfa, "A Novel Decentralized Inter-Vehicle Communications Model Based on Coalition Formation," Journal of Computational Information Systems (JCIS), vol. 10, (2014).

[17].W. Chen, R. K. Guha, T. J. Kwon, J. Lee, and Y. Y. Hsu, "A survey and challenges in routing and data dissemination in vehicular ad hoc networks," Wireless Communications and Mobile Computing, vol. 11, pp. 787-795, (2011). 
[18].K. Kamini and R. Kumar, "Vanet parameters and applications: A review," Global Journal of Computer Science and Technology, vol. 10, (2010).

[19].D. Niyato, Z. Han, W. Saad and A. Hjorungnes, "A controlled coalitional game for wireless connection sharing and bandwidth allocation in mobile social networks," in Global Telecommunications Conference (GLOBECOM 2010), 2010 IEEE, (2010), pp. 1-5.

[20].D. Jiang and L. Delgrossi, "IEEE 802.11 p: Towards an international standard for wireless access in vehicular environments," in Vehicular Technology Conference, 2008. VTC Spring 2008. IEEE, (2008), pp. 2036-2040.

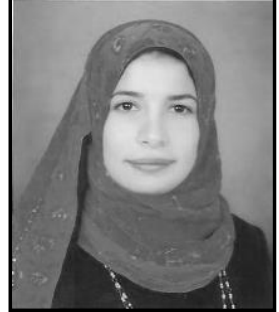

Esraa M. Eldesouky, she is a Ph.D. student in college of Information Science and Engineering at Hunan University. She earned the M.Sc. degree in computer science and technology from Hunan University (2011). She worked at Faculty of Computers and Informatics in Suez Canal University as a teaching assistant from 2005 to 2008. The current research interest includes VANETs, content handling, intelligent transportation systems, and coalition formation game.

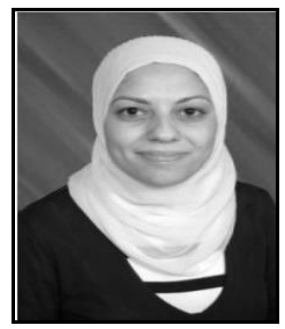

Walaa Elashmawi, she was born in Egypt/1980. She received her B.Sc. from Suez Canal University, Faculty of Computers and Informatics. She got the M.Sc. preliminary from Ain Shams University, Faculty of Computers and Information, and the M.Sc. from Minufiya University in 2008, and the Ph.D from Hunan University, College of Information and Engineering in 2013. She is a Lecturer at Faculty of Computers and Informatics, Suez Canal University. Her research interests include artificial intelligence, multi-agent systems, fuzzy logic, E-commerce system, and computer networks.

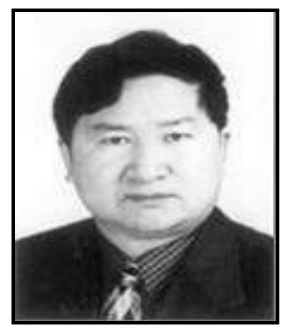

Li Renfa, he is a Professor in the college of Information Science and Engineering at Hunan University. He received the B.Eng. and M. Eng. degrees from Tianjin University, China in 1982 and 1987, and the Ph.D. degree from Huazhong University of Sciences and Technology, China in 2003. He was a Professor at Hunan Technology University from 1987 to 1999. From 2000, he became the dean at the college of Computer and Communication, Hunan University. His research interests are in the areas of embedded system architecture, cyber-physical system and wireless networks. He is the founder of Embedded Systems \& Networking Laboratory of Hunan University, and the leader of Hunan Provincial Key Laboratory of Network and Information Security of Hunan University.

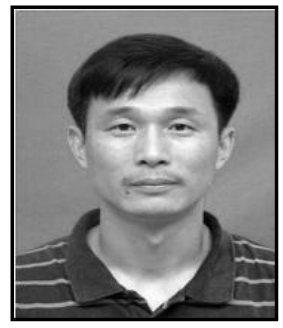

Wang Dong, he was born in 1964. He is a Professor, Ph.D. supervisor of Hunan University. Senior member of China Computer Federation, He has visited University Technology of Sydney, Sydney, Australian from 2004.12 to 2005.12, His current research interests include network test and performance evaluation, wireless communications and mobile computing, VANET etc. He had published more than 70 journal papers. 